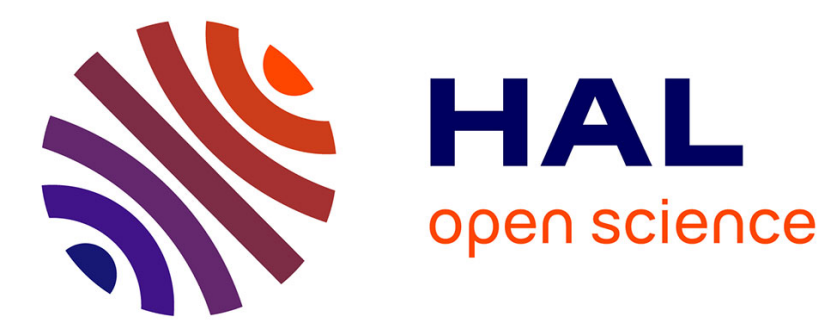

\title{
Transverse phase diffusion in Rayleigh-Bénard convection
}

P. Manneville, J.M. Piquemal

\section{To cite this version:}

P. Manneville, J.M. Piquemal. Transverse phase diffusion in Rayleigh-Bénard convection. Journal de Physique Lettres, 1982, 43 (8), pp.253-258. 10.1051/jphyslet:01982004308025300 . jpa-00232042

\section{HAL Id: jpa-00232042 https://hal.science/jpa-00232042}

Submitted on 1 Jan 1982

HAL is a multi-disciplinary open access archive for the deposit and dissemination of scientific research documents, whether they are published or not. The documents may come from teaching and research institutions in France or abroad, or from public or private research centers.
L'archive ouverte pluridisciplinaire HAL, est destinée au dépôt et à la diffusion de documents scientifiques de niveau recherche, publiés ou non, émanant des établissements d'enseignement et de recherche français ou étrangers, des laboratoires publics ou privés. 
Classification

Physics Abstracts

47.25

\title{
Transverse phase diffusion in Rayleigh-Bénard convection
}

\author{
P. Manneville and J. M. Piquemal \\ SPSRM, CEN Saclay, B.P. No 2, 91191 Gif sur Yvette Cedex, France
}

(Reçu le 25 janvier 1982, accepté le 25 février 1982)

\begin{abstract}
Résumé. - Par un développement formel en gradient de la variable de phase nous obtenons les équations qui gouvernent la relaxation des zig-zags. L'application a été développée dans le cas de la convection près du seuil au moyen d'un développement en $\varepsilon$ de la solution avec condition aux limites de non-glissement pour la vitesse. Les valeurs numériques qui interviennent dans $D_{\perp}$ sont données dans le texte. Les points de comparaison avec d'autres théories et les résultats antérieurs concernant l'instabilité zig-zag sont discutés.
\end{abstract}

\begin{abstract}
By a formal expansion in gradient of the phase variable we obtain the equations governing the relaxation of zig-zags. The application has been developed for convection close to the threshold. We use an $\varepsilon$-expansion of the solution for the realistic case of no slip (rigid) velocity boundary conditions. We obtain :

$$
D_{\perp}=\frac{\xi_{0}^{2}}{\tau_{0}}\left(\frac{\delta k}{k_{\mathrm{c}}}+F(\operatorname{Pr}) \frac{\delta R a}{R a^{\mathrm{c}}}\right)
$$

where the numerical values of the different quantities are given in the text. Points of comparison with other theories and earlier results concerning zig-zag instability are discussed.
\end{abstract}

1. Introduction. - By many aspects Rayleigh-Bénard convection presents itself as a model problem in the field of instability phenomena and dissipative structures. As such it has been the subject of many thorough investigations both theoretical and experimental, the stress being put recently on the problem of the transition to turbulence. However, despite an apparent simplicity of the instability mechanisms at threshold, the behaviour above the threshold is still far from completely understood. To take but only two examples related to the subject of this paper, let us mention the problem of pattern selection which has received various answers (selection through stability [1], boundary effects [2], curvature effects [3]) and the problem of the origin of the low frequency noise close to the threshold in helium [4] which has not yet a clear cut explanation (filtered extrinsic noise [5], intrinsic phase turbulence due to instability [6] or frustration [3], motion of defects [7]). Below we shall face the problem of the analytical determination of phase diffusion coefficients of a convective Boussinesq fluid layer. More precisely we shall study the relaxation of a zig-zag distortion which is the most " dangerous " instability mode close to the threshold. This is not a novel problem. However, up to now most complete results rest either on a Galerkin approximation [8] or on analytical results using Rayleigh's original assumption of free boundary conditions (b.c.) [9-11]. Unfortunately, though it is very popular, this approximation is inadequate to account for usual experimental conditions. Consequences are twofold. First they may be physical : the restoration of (realistic) rigid b.c. strongly inhibits Busse's oscillations 
in low Prandtl number fluids [8]. Second and more crucially, they are mathematical. As a matter of fact, only one mode accounts for the vertical dependence of the linearly unstable solution while three should be necessary for the "generic " case. This accidental peculiarity makes the calculations simpler [12] but it also makes the numerical dependence of most quantities unreliable for a comparison with experiments.

For the case of rigid b.c. apart from numerical results [8] theoretical knowledge is restricted to order $\left(R a-R a^{\mathrm{c}}\right)^{1 / 2}$ and the infinite Prandtl number limit [13a] or more generally but at a qualitative level $[13 b]$. Motivated by all the points evoked above we have undertaken the analytical study of zig-zag diffusion in the vicinity of the threshold for rigid b.c. The derivation closely follows the one developed in reference [6] on a simplified model of convection. Here we only present the formal part of the calculation. The application has been performed using a perturbation expansion [12,13] the details of which will be reported elsewhere [14]. Our results extend those of Schlüter, Lortz and Busse [13] and confirm the findings of Clever and Busse [8] for the borderline of the zig-zag instability. Then we briefly discuss the advantages of the analytical calculation relative to the Galerkin method and also some implications of our results concerning the problems alluded to at the beginning.

2. Determination of the diffusion coefficient. - Due to translational invariance in an infinite fluid layer [15], the position of a system of perfect rolls parallel to the $y$-axis still depends on a phase variable $\varphi$. When $\varphi$ is not a pure constant it has a non-trivial dynamics. Here we restrict ourselves to the case when $\varphi$ depends on the coordinate $y$ along the roll axis. As usual we assume that the fluid motions are described by the Boussinesq equations [1] that we write formally :

$$
\partial_{t} \mathrm{U}=\mathfrak{L}(\mathcal{U})
$$

with $U=\{\mathbf{V}, p, \theta\}$ where $\mathbf{V}=(u, v, w), p$ and $\theta$ are the velocity, pressure and temperature fluctuations respectively. More explicitly the Boussinesq equations read :

$$
\begin{gathered}
\operatorname{div} \mathbf{V}=0 \\
\partial_{t} \mathbf{V}+\mathbf{V} \cdot \operatorname{grad} \mathbf{V}=-\operatorname{grad} p+\operatorname{Pr}[\Delta \mathbf{V}+\mathbf{z} \theta] \\
\partial_{t} \theta+\mathbf{V} \cdot \operatorname{grad} \theta=\Delta \theta+\operatorname{Ra} \mathbf{V} . \mathbf{z} .
\end{gathered}
$$

Let $\mathrm{U}^{0}$ be a 2-dimensional non-linear solution above the threshold. It is a function of $z$ and $x$, periodic in the $x$-direction with period $\lambda=2 \pi / k$. Then $\mathcal{U}^{0}(x+\varphi, z)$ is also a solution when $\varphi$ is constant. If $\varphi$ is slowly varying in space and time $\varphi=\varphi(x, y, t), \mathcal{U}^{0}$ is only an approximate solution which has to be corrected by terms of the order of the gradients of $\varphi$. Replacing $U$ by $\mathcal{u}^{0}+\varphi \partial_{x} \mathcal{u}^{0}+\mathcal{u}^{1}+\mathcal{u}^{2}$ where $\mathcal{u}^{1} \sim \mathcal{O}(\nabla \varphi), \mathcal{u}^{2} \sim \mathcal{O}(\nabla \nabla \varphi) \ldots$ in the Boussinesq equations, one gets a hierarchy of systems to be solved order after order in the formal expansion parameter $\nabla$.

Restricting ourselves to a phase which depends spatially on $y$ only (zig-zag) and assuming $\mathcal{U}^{1}=\partial_{y} \varphi \overrightarrow{\mathrm{U}}^{1}$, at first order we get :

$$
\begin{gathered}
\partial_{y} \varphi\left[\partial_{x} \bar{u}^{1}+\partial_{z} \bar{w}^{1}\right]=0 \\
\partial_{t} \varphi \partial_{x} u^{0}+\partial_{y} \varphi\left[u^{0} \partial_{x} \bar{u}^{1}+\bar{u}^{1} \partial_{x} u^{0}+w^{0} \partial_{z} \bar{u}^{1}+\bar{w}^{1} \partial_{z} u^{0}+\partial_{x} \bar{p}^{1}-\operatorname{Pr}\left(\partial_{x^{2}}+\partial_{z^{2}}\right) \bar{u}^{1}\right]=0 \\
\partial_{t} \varphi \partial_{x} w^{0}+\partial_{y} \varphi\left[u^{0} \partial_{x} \bar{w}^{1}+\bar{u}^{1} \partial_{x} w^{0}+w^{0} \partial_{z} \bar{w}^{1}+\bar{w}^{1} \partial_{z} w^{0}+\right. \\
\left.\quad+\partial_{z} \bar{p}^{1}-\operatorname{Pr}\left(\partial_{x^{2}}+\partial_{y^{2}}\right) \bar{w}^{1}-\operatorname{Pr} \bar{\theta}^{1}\right]=0 \\
\partial_{t} \varphi \partial_{x} \theta^{0}+\partial_{y} \varphi\left[u^{0} \partial_{x} \bar{\theta}^{1}+\bar{u}^{1} \partial_{x} \theta^{0}+w^{0} \partial_{z} \bar{\theta}^{1}+\bar{w}^{1} \partial_{z} \theta^{0}-\left(\partial_{x^{2}}+\partial_{z^{2}}\right) \bar{\theta}^{1}-\operatorname{Ra} \bar{w}^{1}\right]=0
\end{gathered}
$$


that we can write formally :

$$
\partial_{t} \varphi \partial_{x} \mathcal{u}^{0}=\partial_{y} \varphi \frac{\delta \mathcal{L}}{\delta u^{0}} \bar{u}^{1}
$$

where $\delta \mathcal{L} / \delta u^{0}$ stands for $\mathcal{L}$ linearized around $\mathcal{U}^{0}$. A last equation which gives the velocity along $y$ is not coupled with the preceding ones :

$$
\partial_{y} \varphi\left[u^{0} \partial_{x} \bar{v}^{1}+w^{0} \partial_{z} \bar{v}^{1}+\partial_{x} p^{0}-\operatorname{Pr}\left(\partial_{x^{2}}+\partial_{z^{2}}\right) \bar{v}^{1}\right]=0 .
$$

Since we assume $\varphi$ to be a slowly varying function of $y$ and $t, \partial_{y} \varphi$ and $\partial_{t} \varphi$ can be considered as constant with respect to the rapidly varying functions $\mathcal{U}^{0}$ or $\partial_{x} \mathcal{U}^{0}$ so that we have to solve

$$
\frac{\delta \mathcal{L}}{\delta \mathcal{U}^{0}} \overline{\mathcal{W}}^{1}=\partial_{x} \mathcal{U}^{0} \times\left(K=\partial_{t} \varphi / \partial_{y} \varphi\right)=\overline{\mathfrak{F}}^{1} .
$$

For $\bar{u}^{1}$ to exist we have to fulfil the existence condition $\left(\mathcal{U}^{+}, \overline{\mathscr{F}}^{1}\right)=0$ where $U^{+}$is the kernel of the ajoint to $\delta \mathfrak{L} / \delta \mathcal{U}^{0}[16]$. But $\partial_{x} \mathcal{u}^{0}$ is the kernel of $\delta \mathfrak{L} / \delta \mathcal{U}^{0}$ and $\mathcal{U}^{+}$cannot be orthogonal to $\partial_{x} u^{0}$ so that the existence condition imposes $K=0$ i.e. $\partial_{t} \varphi$ of higher order than $\partial_{y} \varphi \cdot \bar{u}^{1}$ can be taken equal to zero except for $\vec{v}^{1} \equiv u^{0}$ as can be easily deduced from the comparison between equation (2e) and the $x$ component of $(1 b)$ for a stationary solution $\left(\partial_{t} \mathbf{V}^{0} \equiv 0\right)$. The physical significance of this result is merely the rotational invariance of the Boussinesq equation. Indeed at order $\partial_{y} \varphi$ the correction to the velocity field is exactly the one necessary to pass from rolls aligned parallel to the $y$-axis to infinitesimally tilted rolls.

At order $\partial_{y^{2}}$ the system reads :

$$
\partial_{t} \varphi \partial_{x} u^{0}=\partial_{y^{2}} \varphi\left[\frac{\delta \mathfrak{L}}{\delta \mathcal{U}^{0}}{\overline{u^{2}}}^{2}+\overline{\mathcal{G}}^{2}\right]
$$

where $\overline{\mathrm{\Phi}}^{2}=\left\{-u_{0},-\operatorname{Pr} \partial_{x} u_{0},-\operatorname{Pr} \partial_{x} w^{0},-\partial_{x} \theta^{0}\right\}$ the equation giving $\bar{v}^{2}$ has been omitted since it does not contribute. Now we assume that $\partial_{t} \varphi$ is of the order of $\partial_{y^{2}} \varphi$ through the definition of the transverse diffusion coefficient $D_{\perp}$ :

$$
\partial_{t} \varphi=D_{\perp} \partial_{y^{2}} \varphi
$$

and we get $D_{\perp}$ in writing the existence condition for the solution at order 2 :

$$
D_{\perp}\left(\mathcal{u}^{+}, \partial_{x} \mathcal{u}^{0}\right)=\left(\mathcal{u}^{+}, \mathcal{G}^{2}\right) .
$$

Up to now this is totally general. To be specific, we have to inject a non-linear solution $\mathcal{u}^{0}$. This could be a solution obtained by a Galerkin approximation [8]; here we have chosen to substitute the solution of the $\varepsilon$-expansion $[12,13]$. For that purpose we have solved the nonlinear stationary solution of the Boussinesq equations to order 3 in $\varepsilon$ with rigid b.c. recovering all numerical results of reference [13] but getting in addition the complete solution at order $\varepsilon^{3}$ as a function of the Prandtl number, which turns out to be necessary to get $D_{\perp}$ at order

$$
\varepsilon^{2} \sim \delta R a=R a-R a^{\mathrm{c}} .
$$

$D_{\perp}$ is a priori a function of the distance to the threshold and of the wave vector of the solution at order zero, $\delta k=k-k_{\mathrm{c}}$. Thus we look for $D_{\perp}$ under the form of a double expansion :

$$
D_{\perp}=D_{00}+D_{01} \delta k+D_{10} \varepsilon+D_{20} \varepsilon^{2}+\cdots
$$

By a manipulation of the integrals which express the existence condition at order $\varepsilon$ one can 
show that $D_{00} \equiv 0$ and that $D_{01}=\xi_{0}^{2} / \tau_{0} k_{\mathrm{c}}$, where $\xi_{0}$ and $\tau_{0}$ are the coherence length and relaxation time of the structure far from the threshold [17]. $D_{10} \equiv 0$ results from the fact that it is the only term with wave vector $k_{\mathrm{c}}$ in the equations at order $\varepsilon^{2}$ in factor of a set of functions not orthogonal to the kernel of the linear operator. The computation of $D_{20}$ is quite involved and will be presented in details elsewhere [14].

Using $\delta R a=R a-R a^{\mathrm{c}}=R_{2}(\operatorname{Pr}) \varepsilon^{2}$, the result can be recast as

$$
D_{\perp}=\frac{\xi_{0}^{2}}{\tau_{0}}\left(\frac{\delta k}{k_{\mathrm{c}}}+\frac{N(P r)}{R_{2}(P r)} \frac{\delta R a}{R a^{\mathrm{c}}}\right)
$$

$\xi_{0}^{2} / \tau_{0}$ being obviously the right combination for a diffusion coefficient. Numerically for rigidrigid velocity b.c. we get :

$$
\begin{aligned}
R a^{\mathrm{c}} & =1707.762 \quad k_{\mathrm{c}}=3.11632 \\
\xi_{0}^{2} / \tau_{0} & =5.6851 /(1.9545+1 / P r) \\
N(P r) & =0.16591+23.0395 / P r+6.1961 / \operatorname{Pr}^{2} \\
R_{2}(P r) & =10.75796-0.07256 / P r+0.12805 / \operatorname{Pr}^{2} .
\end{aligned}
$$

(The expression for $R_{2}$ can be found in reference [13a] with a different normalization which amounts to a multiplication by $q_{c}^{4}$.)

3. Discussion. - Due to rotational invariance of the Boussinesq equations in an infinite layer, an infinitesimal rotation of the rolls is expected not to relax. Thus zig-zags which are long wavelength modifications of a uniform rotation are expected to be very long lived. The associated diffusion coefficient is very small, but may be positive for stability or negative for instability. The curve $D_{\perp}=0$ corresponds to neutrality against a slight curvature. Zig-zags are thus the most "dangerous " modes for supercritical convection. The frontier for instability is easily deduced from (5).

At order $\sqrt{\delta R a}, D_{\perp}$ reduces itself to $\frac{\xi_{0}^{2}}{\tau_{0}} \times \frac{\delta k}{k_{\mathrm{c}}}$. Thus we recover the analytical result of Schlüter, Lortz and Busse that the structure is unstable for $\delta k<0$ [18]. To the following order the unstable domain in the plane $(\delta k, \delta R a)$ is on the left of a curve which starts as

$$
\frac{\delta R a}{R a^{\mathrm{c}}}=-Q(\operatorname{Pr}) \frac{\delta k}{k_{\mathrm{c}}}
$$

It is easily seen that $Q(P r)=R_{2}(P r) / N(P r)$ is always positive so that the slope is always negative [24]. As observed by Clever and Busse, the curve rotates counterclockwise when $\operatorname{Pr}$ decreases. For $\operatorname{Pr}=\infty$ we get $Q \sim 65$ while for $\operatorname{Pr}=7$ and 0.7 we get $Q \sim 3$ and $\sim 0.24$ respectively, in good agreement with results in references [8] and [19]. At even smaller Prandtl number the zig-zag instability does not disappear but the slope of its boundary is very small $Q \rightarrow 0.02$ for $\mathrm{Pr} \rightarrow 0$; this limiting behaviour is probably out of reach of the Galerkin approximation. The main advantage of the analytical calculation over the Galerkin method is that one can easily isolate the different contributions to $D_{\perp}$. As a matter of fact in (3) one has two kinds of terms, those issuing directly from the unperturbed solution $\left(\overline{\mathcal{G}}^{(2)}\right)$ and those emerging from the expansion of $\frac{\delta \mathcal{L}}{\delta U^{(0)}} \mathcal{U}^{(2)}$. Among these last terms the most important one is an $x$-velocity component which depends on $z$ but not on $x$. Convection of the temperature field $\theta^{(0)}$ by this component gives the major contribution of the $1 / P r$ term in $N(P r)$ while convection of the velocity field $\left(u^{(0)}, w^{(0)}\right)$ is mostly responsible for the $1 / P r^{2}$ term. This observation gives a concrete meaning to the often quoted "stabilizing effect of convective terms". 
The main drawback of the analytical calculation is its limited validity range. At infinite Prandtl number, a comparison [20] between the $\varepsilon$-expansion, the Galerkin method and the experiments shows that the basic mode is overestimated by the second method and that the first one should not be extrapolated too far and this is confirmed by our analysis of higher order terms of the $\varepsilon$-expansion which suggests that it has a finite radius of convergence which decreases with $\operatorname{Pr}[14]$.

The derivation of the phase diffusion equation is a multiple scale method analogous to that for the well known amplitude equation [10]. The difference is that from the beginning one gets rid of amplitude variations. This allows to get relatively easily non-trivial results (at least at the formal level, for the numerical application to a particular case - here rigid b.c. - can be quite involved). Despite this limitation, our result has some importance since it has been recognized recently [7] that it is this constant amplitude part of the deformation around a dislocation which governs the velocity of the defects, according to a law of the form $v \sim D_{\perp}^{3 / 2}$.

As another check of our theory, one can look for Busse's oscillation mode [21] since our derivation is very similar to his expansion in powers of the wave vector $k_{y}$. In fact it is easy to see that assuming free b.c. one can get at order $\partial_{y} \varphi$ a special solution $\overline{\bar{u}}^{1} \sim-K\left(=-\partial_{t} \varphi / \partial_{y} \varphi\right)=$ const. where $K$ is nothing else but $\sigma_{1}$ in the expansion $\sigma=\sigma_{1} k_{y}+\sigma_{2} k_{y}^{2}+\cdots$ of the relaxation rate of the perturbation. At second order in $\hat{c}_{y}$ a first condition fixes $\overline{\bar{u}}^{1}$ at the value predicted by Busse and one has to introduce a second constant $\overline{\bar{u}}^{2}$; the existence condition no longer fixes $D_{\perp}$ but a combination involving $\sigma_{2}$ and $\overline{\bar{u}}^{2}, \overline{\bar{u}}^{2}$ being determined at order 3 in $\partial_{y}$. In order to recover the (non-oscillating) zig-zag instability one has to force $\overline{\bar{u}}^{1}$ to zero and to compensate constant terms in the equation for $\bar{u}^{2}$ by a constant pressure gradient $\partial_{x} \overline{\bar{p}}^{2}$. Doing this one gets the known value $D_{\perp}=\frac{4 \operatorname{Pr}}{1+\operatorname{Pr}} \frac{\delta k}{k_{\mathrm{c}}}$ at order $\sqrt{\delta R a} \cdot \overline{\bar{u}}_{1}$ corresponds to the field $B_{x}$ introduced by Siggia and Zippelius [11] in their generalized amplitude equation. However two points must be stressed. The first one is that these constants $\overline{\bar{u}}_{1}, \overline{\bar{u}}_{2}, \ldots$ are only allowed for free and forbidden by rigid b.c. The second point is that even staying with unrealistic free b.c. one seems to face an alternative. Let $\operatorname{Pr}$ kept constant and assume $\varepsilon$ given. Either one assumes $\overline{\bar{u}}_{1}, \overline{\bar{u}}_{2}, \ldots=0$ and one gets zig-zags or $\overline{\bar{u}}_{1}, \overline{\bar{u}}_{2}, \ldots \neq 0$ and the oscillations emerge. But for $\varepsilon$ small enough, rolls are stable against oscillations since one must have $\varepsilon \gtrsim \operatorname{Pr}$ for sustained oscillations [21] and one is left only with the zig-zag instability for $\varepsilon \rightarrow 0$. A similar alternative remains for rigid b.c., the consequences of which are yet unclear as to the role of the couplings introduced by Siggia and Zippelius [11].

The concept of phase diffusion in convective structures has already been the subject of experimental checks [22]. For phase diffusion parallel to the wave vector of the zeroth order solution, the amplitude equation at lowest order is already sufficient [10]. This is no longer the case for transverse phase diffusion. By-passing the derivation of a higher order amplitude equation, our result brings a non-trivial answer. The next steps would be to study the problem of structure selection and defect motions using similar analytic tools with realistic boundary conditions. This would certainly give valuable information on the birth of turbulence close to the convection threshold in large aspect ratio containers and more especially in moderate and low Prandtl number [4].

Acknowledgments. - The authors would like to thank V. Croquette, Y. Pomeau and E. Weisfreid for interesting discussions related to this work and for the communication of certain results prior to publication.

\section{References}

[1] Busse, F. H., Rep. Prog. Phys. 41 (1978) 1929.

[2a] Cross, M. C., Daniels, P. G., Hohenberg, P. C., Siggia, E. D., Phys. Rev. Lett. 45 (1980) 898 and to appear. 
[2b] Pomeau, Y., Zaleski, S., J. Physique 42 (1981) 515.

[3] Pomeau, Y., Manneville, P., J. Physique 42 (1981) 1067.

[4a] Ahlers, G., Behringer, R. P., Phys. Rev. Lett. 40 (1978) 712.

[4b] Libchaber, A., Maurer, J., J. Physique-Lett. 39 (1978) L-369.

[5] Greenside, H. S., Ahlers, G., Hohenberg, P. C., Walden, R. W., Bell Labs. Preprint (1981).

[6] Pomeau, Y., Manneville, P., J. Physique-Lett. 40 (1979) L-609.

[7] Siggia, E. D., Zippelius, A., Phys. Rev. A 24 (1981) 1036.

[8] Clever, R. M., Busse, F. H., J. Fluid. Mech.65(1974) 625.

[9] Joseph, D. D., Stability of Fluid Motion II, Ch. XI, Springer Tracts in Natural Philosophy, Vol. 28 (Springer-Verlag, N.Y.) 1976.

[10] Newell, A. C., WhiteheAd, J. A., J. Fluid Mech. 38 (1979) 279.

[11] Siggia, E. D., Zippelius, A., Phys. Rev. Lett. 47 (1981) 835.

[12] Malkus, W. V. R., Veronis, G., J. Fluid. Mech. 4 (1958) 225.

[13a] SCHLÜter, A., LorTZ, D., Busse, F., J. Fluid. Mech. 23 (1965) 129.

[13b] Busse, F. H., in Instability of Continuous Systems, H. Leipholtz ed. (Springer-Verlag, New York) 1971.

[14] Piquemal, J. M., Thèse de 3e cycle, Université de Paris-Vi (1982) and Manneville, P., Piquemal, J. M., to be submitted for publication in J. Physique.

[15] The limit of the infinite medium is never achieved in practice however for short enough durations or sufficiently rapid dynamical phenomena, the system does not feel its boundaries and behaves as if it were infinite; see SnedDon, L., Phys. Rev. A 24 (1981) 1629.

[16] Coddington, E. A., Levinson, N., Theory of ordinary differential equations (Mc Graw-Hill, New York) 1955.

[17] Weisfreid, J., Pomeau, Y., Dubois, M., Normand, C., Bergé, P., J. Physique 39 (1978) 725.

[18] There is systematically a difference of 1 between the order at which the solution is known and the order at which $D_{\perp}$ is determined. This explains the apparent discrepancy between reference [13] and the present formulation. In reference [13] stability of the solution was determined at order $\varepsilon^{2}$ only, which allows to understand their misinterpretation of stability boundaries (see their Fig. 1).

[19] Clever, R. M., Busse, F. H., J. Fluid. Mech. 102 (1981) 61.

[20] Dubois, M., Normand, C., Bergé, P., Int. J. Heat Mass Transfer 21 (1978) 999.

[21] Busse, F. H., J. Fluid. Mech. 52 (1972) 97.

[22] Weisfreid, J. E., CroQuette, V., Phys. Rev. Lett. 45 (1980) 634.

[23] Croquette, V., Schosseler, F., submitted to J. Physique.

[24] Such an expression has already been given by Busse (Ref. [13b]) who did not calculate the quantity $Q(P r)$ thus leaving open the question of its sign. 